\title{
Subspace-Based Channel Estimation for Code Division Multiple Access Communication Systems
}

\author{
Stephen E. Bensley and Behnaam Aazhang, Senior Member, IEEE
}

\begin{abstract}
We consider the estimation of channel parameters for code-division multiple access (CDMA) communication systems operating over channels with either single or multiple propagation paths. The multiuser channel estimation problem is decomposed into a series of single user problems through a subspace-based approach. By exploiting the eigenstructure of the received signal's sample correlation matrix, the observation space can be partitioned into a signal subspace and a noise subspace without prior knowledge of the unknown parameters. The channel estimate is formed by projecting a given user's spreading waveform into the estimated noise subspace and then either maximizing the likelihood or minimizing the Euclidean norm of this projection. Both of these approaches yield algorithms which are near-far resistant and do not require a preamble.
\end{abstract}

\section{INTRODUCTION}

$\mathbf{I}$ $\mathrm{N}$ a code-division multiple access (CDMA) communication system, all users simultaneously occupy the same frequency band, and thus great care must be taken to limit multipleaccess interference (MAI). Although the spreading waveforms are designed with low cross-correlations, the users' signals are not truly orthogonal. If power levels are widely varying, code design alone may be insufficient for suppressing MAI. This is the near-far problem.

Conventional CDMA systems either ignore the near-far problem or try to limit it with power control, and thus, standard single user techniques (e.g., matched filters, active correlators, state estimators, etc.) are used for synchronization and detection [1]. However, even a small amount of the near-far effect can drastically degrade the performance of conventional receivers [2]. For many years, this was thought to be an inherent limitation of CDMA until Verdú developed the optimum multiuser detector [3]. Verdú's work was followed by many suboptimal schemes of lower computational complexity [4]-[6], all of which are near-far resistant. However, these methods deal only with detection and assume that the timing of the spreading waveforms is known.

In addition, many wireless transmission channels are characterized by multiple propagation paths that further complicate

Paper approved by G. L. Stiiber, the Editor for Spread Spectrum of the IEEE Communications Society. Manuscript received January 9, 1995; revised August 18,1995 . This work was supported in part by the National Aeronautics and Space Administration under Grant NGT-51050 and in part by the Advanced Technology Program of the Texas Higher Education Coordinating Board under Grant 003604-018. This paper was presented in part at the 1994 IEEE International Symposium on Information Theory, Trondheim, Norway, and in part at the Communication Theory Mini-Conference at IEEE GLOBECOM '94, San Francisco, CA.

The authors are with the Department of Electrical and Computer Engineering, Rice University, Houston, TX 77251-1892 USA.

Publisher Item Identifier S 0090-6778(96)06314-3. synchronization. This problem is particularly severe in urban and indoor environments [7], [8], which are of great interest for cellular mobile radio applications and wireless local area networks. Direct sequence spread spectrum (DS/SS) has often been studied for these applications; however, many radio channels exhibit such severe multipath effects that even the strict autocorrelation properties of the spreading waveforms are inadequate for rejecting multipath interference. Furthermore, rather than simply rejecting multipath, we can exploit this time diversity by using a RAKE receiver matched to each propagation path [9], thus capturing the multipath energy and improving our overall signal-to-noise ratio (SNR). The multiuser detection problem has been extended to multipath channels [10], [11], where it has been shown that the outputs of a bank of filters matched to each propagation path for each user (or equivalently a RAKE correlator for each user) form the sufficient statistics. However, to optimally "recombine" the multipath requires knowledge of the relative delays, amplitudes, and phases of the various propagation paths.

Most of the work done on timing acquisition for CDMA systems focuses on jointly estimating the necessary parameters for all users [11]-[13]. While these techniques produce excellent results, they can be computationally intense since they involve solving a multidimensional optimization problem for a large number of parameters.

In [14], the authors presented a technique for reducing this to a one-dimensional (1-D) problem while retaining the performance of maximum likelihood estimation. This method, however, requires a preamble and is incapable of multiuser timing estimation. Subspace-based techniques avoid both of these drawbacks, while providing a straightforward method for decomposing a multidimensional parameter search into a series of 1-D optimization problems. Subspace-based estimation has been widely studied for array signal processing, system identification, and time series analysis (see [15] and references within). The received signal is modeled as an arbitrary linear combination of several unknown signal vectors plus noise. These signal vectors are known functions of unknown parameters that we would like to estimate. By exploiting the eigenstructure of the correlation matrix, the observation space can be partitioned into a signal subspace and a noise subspace without prior knowledge of the signal vectors. In this paper, we examine a CDMA network within this framework, where the signal vectors are the spreading waveforms and the unknown parameters are the channel 
coefficients. ${ }^{1}$ The estimate is based on the projection of a given user's signal vectors into the estimated noise subspace.

\section{ANALyTICAL MOdel of a CDMA System}

\section{A. Spread Spectrum Signal}

In a CDMA network, several users transmit simultaneously over a common channel, so the received signal can be modeled as the superposition of $K$ active users with additive channel noise

$$
r(t)=\sum_{k=1}^{K} r_{k}(t)+\eta_{t} \quad-\infty<t<\infty
$$

where $\eta_{t}$ is assumed to be white Gaussian noise with zero mean and a two-sided power spectral density of $\mathcal{N}_{0} / 2$. If the transmission channel is linear, then $r_{k}(t)$ can be expressed as the convolution of the transmitted signal $s_{k}(t)$ with the time-varying channel impulse response $h_{k}(\tau, t)$

$$
\begin{aligned}
r_{k}(t) & =h_{k}(t, \tau) * s_{k}(t) \\
& =\int_{-\infty}^{\infty} h_{k}(t, \alpha) s_{k}(\alpha) d \alpha .
\end{aligned}
$$

Note, that in a multiuser system, a different channel impulse response may be associated with each user's signal since in general the users are transmitting from different locations. In a cellular system, this will be the case for the reverse link, whereas the channel impulse response will be the same for the forward link.

If phase-shift keying (PSK) is used to modulate the data, then the baseband complex envelope representation of the $k$ th user's transmitted signal is given by

$$
s_{k}(t)=\sqrt{2 P_{k}} e^{j \phi_{k}} \sum_{i} e^{j(2 \pi / M) m_{k}^{(i)}} a_{k}(t-i T)
$$

where $P_{k}$ is the transmitted power, $\phi_{k}$ is the carrier phase relative to the local oscillator at the receiver, $M$ is the size of the symbol alphabet, $m_{k}^{(i)} \in\{0,1, \cdots, M-1\}$ is the transmitted symbol, $a_{k}(t)$ is the spreading waveform, and $T$ is the symbol duration.

The spreading waveform $a_{k}(t)$ is formed by modulating a signature sequence of $N$ "chips." If PSK is also used for the spreading modulation, then the spreading waveform is given by

$$
a_{k}(t)=\sum_{n=0}^{N-1} \Pi_{T_{c}}\left(t-n T_{c}\right) a_{k}^{(n)}
$$

where $\Pi_{T_{c}}(t)$ is a rectangular pulse, $T_{C}$ is the chip duration $\left(T_{c}=T / N\right)$, and $\left\{a_{k}^{(n)}\right\}$ for $n=0,1, \cdots, N-1$ is a signature sequence (possibly complex valued since the signature alphabet need not be binary).

Since we would like to do our signal processing digitally, the received continuous-time signal is converted into discretetime by sampling the output of a filter matched to the chip waveform. For PSK, the chip waveform is a rectangular pulse,

\footnotetext{
${ }^{1}$ See [16], where the authors report on a similar but independent work.
}

so the matched filter can be implemented as an integrate-anddump circuit, and the discrete-time signal is given by

$$
r[n]=\frac{1}{T_{c}} \int_{n T_{c}}^{(n+1) T_{c}} r(t) d t .
$$

Since the chip timings of the users are unknown, the integration interval of the filter is chosen arbitrarily. Thus, a given interval will generally contain components from two chips for each user. This averaging of adjacent chips attenuates the high frequency components of the spreading waveforms, resulting in an average $\mathrm{e}^{2}$ loss of $1.76 \mathrm{~dB}$ to the SNR with a worst case loss of $3 \mathrm{~dB}$ when the misalignment is exactly $\frac{1}{2} T_{c}$. While this obviously increases the estimation error, in many cases this is preferable to the alternative of sampling faster than the chip rate. ${ }^{3}$

Due to the underlying periodicities of the spreading waveforms, the covariance function of the received signal is not shift-invariant, and thus $r[n]$ is not wide-sense stationary (WSS). This can cause problems since many traditional signal processing techniques are tailored toward WSS random processes. Fortunately, $r[n]$ is wide-sense cyclostationary. Its mean function is zero and thus trivially periodic, and the covariance function is invariant to a shift by $N$ in both its arguments. Thus, the received signal can be converted into a sequence of WSS random vectors by buffering $r[n]$ into blocks of length $N$

$$
\mathbf{y}_{i}=[r[i N] \quad r[1+i N] \quad \cdots r[N-1+i N]]^{\top} \in \mathbb{C}^{N}
$$

where the $n$th element of the $i$ th observation vector is given by $y_{i, n}=r[n+i N]$ defined in (5).

Although each observation vector corresponds to one symbol interval, this buffering was done without regard to the actual symbol intervals of the users. Since the system is asynchronous, each observation vector will contain at least the end of the previous symbol and the beginning of the current symbol for each user (see Fig. 1). Multipath effects may result in additional components from earlier symbols; however, we will assume that the multipath delay spread is small enough that these can be neglected. Thus, each observation vector can be viewed as a linear combination of $2 K$ signal components plus noise. Since for now we are only interested in estimating the channel impulse response, the factors due to the power, phase, and transmitted symbols of the $k$ th user may be collected into a single complex constant $c_{k}^{(i)}$, e.g., some constant times $\sqrt{2 P_{k}} e^{j\left[\phi_{k}+(2 \pi / M) m_{k}^{(i)}\right]}$. The signal model for all $K$ users can now be written as

$$
\mathbf{y}_{i}=\sum_{k=1}^{K}\left[c_{k}^{(i-1)} \mathbf{u}_{k}^{r}+c_{k}^{(i)} \mathbf{u}_{k}^{l}\right]+\boldsymbol{\eta}_{i}=\mathbf{A} \mathbf{c}_{i}+\boldsymbol{\eta}_{i}
$$

where $\boldsymbol{\eta}_{i}=\left[\eta_{i, 0}, \cdots, \eta_{i, N-1}\right]^{\top} \in \mathbb{C}^{N}$ is a Gaussian random vector and its elements are zero mean with variance $\sigma^{2}=$ $\mathcal{N}_{0} / 2 T_{c}$ and are mutually independent. The signal vectors $\mathbf{u}_{k}^{r}$ and $\mathbf{u}_{k}^{l}$ depend only on the user's spreading waveform and

\footnotetext{
${ }^{2}$ The average loss in $\mathrm{dB}$ is calculated as $10 \log 1.5$

${ }^{3}$ The drop in SNR can be reduced by integrating and dumping every $T_{c} / 2$ $s$ instead of every $T_{c}$. Obviously, increasing the sampling rate increases the size of the data and in turn increases the hardware requirements.
} 


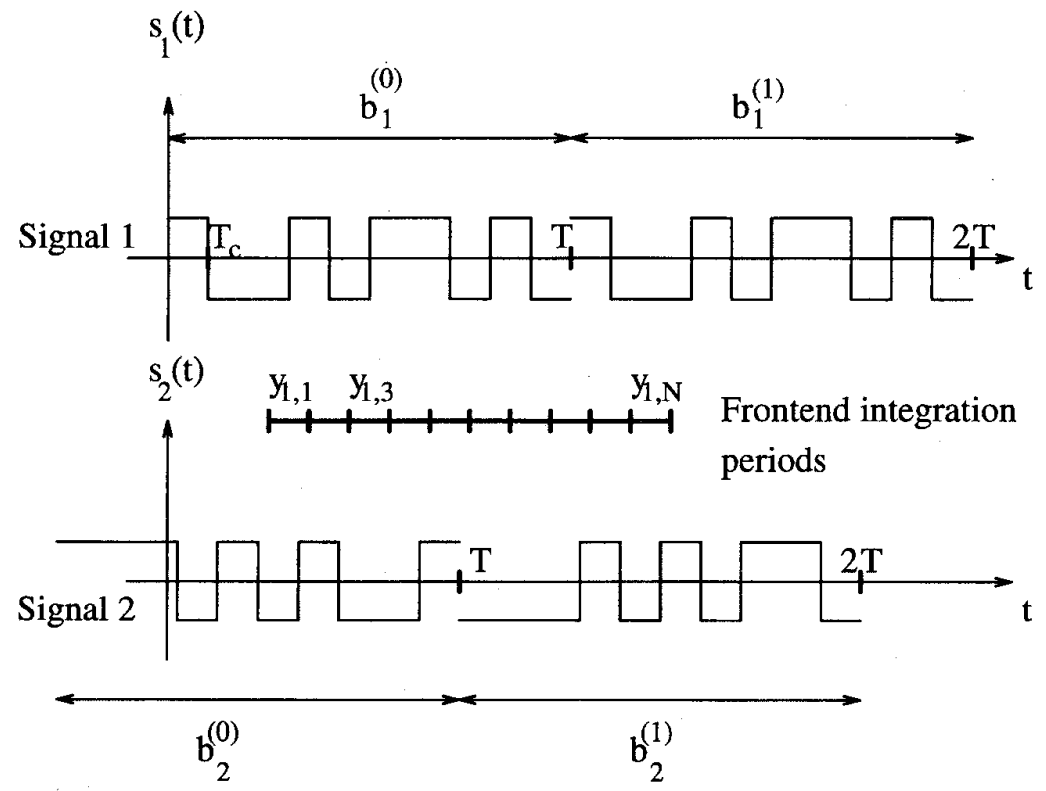

Fig. 1. Diagram to depict a two-user asynchronous system with arbitrary timing of chip integrations. The statistics $y_{i, n}$ are defined in (6). Note that since the users and the receiver are not synchronized, in the integration over one chip period, there are contributions from two adjacent chips for each user.

the associated channel impulse response. Thus, if the channel is time-invariant, the signal vectors do not vary with $i$. It is possible to gain insight from a simple channel model where these vectors $\mathbf{u}_{k}^{r}$ and $\mathbf{u}_{k}^{l}$ are the right side of the $k$ th user's code vector followed by zeros and zeros followed by the left side of the $k$ th user's code vector, respectively. These vectors will be defined more precisely for different channels in the following section. Note that in (7), we have defined $\mathbf{c}_{i}=\left[\begin{array}{lllll}c_{1}^{(i-1)} & c_{1}^{(i)} & \cdots & c_{K}^{(i-1)} & c_{K}^{(i)}\end{array}\right]^{\top} \in \mathbb{C}^{2 K}$ and the signal matrix $\mathbf{A}=\left[\begin{array}{lllll}\mathbf{u}_{1}^{r} & \mathbf{u}_{1}^{l} & \cdots & \mathbf{u}_{K}^{r} & \mathbf{u}_{K}^{l}\end{array}\right] \in \mathbb{C}^{N \times 2 K}$.

An important issue that we will not address is the determination of the number of users. For this paper, it will be assumed that $K$ is known; however, a variety of techniques have been proposed for estimating the model order (e.g., [17]).

\section{B. Transmission Channel}

To examine how the signal vectors depend on the channel impulse response, we first consider a transmission channel where each user's signal goes through a single propagation path with an associated attenuation factor and propagation delay. We assume that these parameters vary slowly with time, so that for sufficiently short intervals the channel is approximately a linear time-invariant (LTI) system. The baseband channel impulse response can then be represented by a Dirac delta function as $h_{k}(t, \tau)=h_{k}(t)=\alpha_{k} \delta\left(t-\tau_{k}\right), \quad \forall \tau$, where $\alpha_{k}$ is a complex valued attenuation weight and $\tau_{k}$ is the propagation delay. Since there is just a single path, we assume that $\alpha_{k}$ is incorporated into $c_{k}^{(i)}$ and concentrate solely on the delay.

By sampling and blocking the input data, the receiver has established an arbitrary timing reference. In order to successfully "despread" a given user and detect its data symbols, we only need to know the chip and symbol timing relative to this reference; thus, the propagation delay $\tau_{k}$ can be evaluated modulo the symbol period $T$.

We now define $\nu \in\{0, \cdots, N-1\}$ and $\gamma \in[0,1)$ such that $\left(\tau_{k} / T_{c}\right) \bmod N=\nu+\gamma$. Let's first consider the case where $\gamma=0$, i.e., the received signal is precisely aligned with the chip matched filter. For this special case, only one chip will contribute to each sample, and the signal vectors are

$$
\begin{aligned}
\mathbf{u}_{k}^{r} & =\mathbf{a}_{k}^{r}(\nu) \\
& \equiv\left[\begin{array}{llllll}
a_{k}^{(N-\nu)} & \cdots & a_{k}^{(N-1)} & 0 & \cdots & 0
\end{array}\right]^{\top} \\
\mathbf{u}_{k}^{l} & =\mathbf{a}_{k}^{l}(\nu) \\
& \equiv\left[\begin{array}{llllll}
0 & \cdots & 0 & a_{k}^{(0)} & \cdots & a_{k}^{(N-\nu-1)}
\end{array}\right]^{\top} .
\end{aligned}
$$

Since the chip matched filter is just an integrator, the samples for a nonzero $\gamma$ will be a convex combination of two adjacent chips

$$
\begin{aligned}
\mathbf{u}_{k}^{r} & =(1-\gamma) \mathbf{a}_{k}^{r}(\nu)+\gamma \mathbf{a}_{k}^{r}(\nu+1) \\
\mathbf{u}_{k}^{l} & =(1-\gamma) \mathbf{a}_{k}^{l}(\nu)+\gamma \mathbf{a}_{k}^{l}(\nu+1) .
\end{aligned}
$$

We now consider the more general case of a multipath transmission channel with $L$ distinct propagation paths. If we retain the LTI approximation, the impulse response becomes a series of delta functions

$$
\begin{aligned}
h_{k}(t, \tau) & =h_{k}(t) \\
& =\sum_{p=1}^{L} \alpha_{k, p} \delta\left(t-\tau_{k, p}\right) .
\end{aligned}
$$

The multipath spread of the channel, $T_{m}$, is the maximum difference between propagation delays, i.e., $T_{m}=$ $\max _{p, p^{\prime}}\left|\tau_{k, p}-\tau_{k, p^{\prime}}\right|$. Typical values for the multipath spread are $0.3 \mu \mathrm{s}$ for indoor channels and $10 \mu \mathrm{s}$ for outdoor channels [7], [8]. We assume that the multipath spread is less than half 
the symbol period $\left(T_{m}<\frac{1}{2} T\right)$, which is consistent with data rates of $1.5 \mathrm{Mbs}$ indoors and $50 \mathrm{Kbs}$ outdoors.

Since the amplitudes and phase shifts of the various paths may be quite different, we can no longer neglect the $\alpha_{k, p}$ 's. Thus, the signal vectors will be a weighted sum of the convex combinations corresponding to each path

$$
\begin{aligned}
\mathbf{u}_{k}^{r}= & \sum_{p=1}^{L} \alpha_{k, p}\left[\left(1-\gamma_{k, p}\right) \mathbf{a}_{k}^{r}\left(\nu_{k, p}\right)\right. \\
& \left.+\gamma_{k, p} \mathbf{a}_{k}^{r}\left(\nu_{k, p}+1\right)\right], \\
\mathbf{u}_{k}^{l}= & \sum_{p=1}^{L} \alpha_{k, p}\left[\left(1-\gamma_{k, p}\right) \mathbf{a}_{k}^{l}\left(\nu_{k, p}\right)\right. \\
& \left.+\gamma_{k, p} \mathbf{a}_{k}^{l}\left(\nu_{k, p}+1\right)\right] .
\end{aligned}
$$

If we define

$$
\begin{aligned}
\mathbf{U}_{k}^{r} & =\left[\begin{array}{lll}
\mathbf{a}_{k}^{r}(0) & \cdots & \mathbf{a}_{k}^{r}(N-1)
\end{array}\right] \in \mathbb{C}^{N \times N} \\
\mathbf{U}_{k}^{l} & =\left[\begin{array}{lll}
\mathbf{a}_{k}^{l}(0) & \cdots & \mathbf{a}_{k}^{l}(N-1)
\end{array}\right] \in \mathbb{C}^{N \times N}
\end{aligned}
$$

where the $a_{k}$ 's are as defined in (8), then the signal vectors may be expressed as a linear combination of the columns of these matrices

$$
\begin{aligned}
& \mathbf{u}_{k}^{r}=\mathbf{U}_{k}^{r} \mathbf{h}_{k} \\
& \mathbf{u}_{k}^{l}=\mathbf{U}_{k}^{l} \mathbf{h}_{k}
\end{aligned}
$$

where $\mathbf{h}_{k}$ is the composite impulse response of the channel and the receiver front-end, evaluated modulo the symbol period. Thus, the $n$th element of the impulse response is given by

$$
h_{k, n}=\sum_{j=0}^{\infty} \frac{1}{T_{c}} \int_{j T+n T_{c}}^{j T+(n+1) T_{c}} h_{k}(t) * \Pi_{T_{c}}(t) d t .
$$

Note that due to our restrictions on the multipath spread, at most two terms in the summation will be nonzero.

\section{Subspace BASED ChanNel Estimation}

\section{A. Subspace Approach}

If the users' signal vectors are linearly independent, the data component of each observation vector $\mathbf{y}_{i}$ will lie in the $2 K$-dimensional subspace spanned by the columns of the signal matrix $\mathbf{A}$. Thus, the observation space may be partitioned into a signal subspace $\left(\mathcal{S}_{Y}\right)$ and a noise subspace $\left(\mathcal{N}_{Y}\right)$. From our model, it is clear that for a given set of $K$ spreading waveforms, there is a well-defined mapping $G$ from the channel parameters to the resulting signal subspace, i.e., $G\left(\left\{\mathbf{h}_{k}\right\}\right)=\mathcal{S}_{Y}$. If this mapping is one-to-one and thus, invertible, then the channel parameters can be uniquely determined from $\mathcal{S}_{Y}$. For our purposes, we will assume that this bijection can be guaranteed by proper choice of codes or by placing restrictions on the class of channels over which we operate.

In the absence of noise, once $2 K$ linearly independent observation vectors have been collected, we can determine $\mathcal{S}_{Y}$ exactly, and the desired parameters will immediately follow. Of course, in practice the observation vectors are corrupted with noise, and we must estimate the signal subspace. Since a parametric approach would be prohibitively complex, we instead form an unconstrained estimate $\hat{\mathcal{S}}_{Y}$, i.e., a set of basis vectors that best fit the observed data without regard to our analytical model for the received signal. While this drastically reduces the complexity of the estimator, the result is that with probability one, $\hat{\mathcal{S}}_{Y}$ will not lie in the range space of $G$, and thus $G^{-1}\left(\hat{\mathcal{S}}_{Y}\right)$ is undefined [18]. Therefore, we must instead choose a feasible $\mathcal{S}_{Y}$ that is "close" in some sense to $\hat{\mathcal{S}}_{\mathrm{Y}}$. While a wide variety of algorithms have been proposed [19], we follow an approach based on the projection of a user's signal vectors into the estimated noise subspace. This method has the nice property of allowing us to form each user's estimate independently of the others, which drastically decreases complexity.

\section{B. Estimating the Signal Subspace}

The correlation matrix of the observation vectors is given by

$$
\begin{aligned}
\mathbf{R} & =E\left[\mathbf{y}_{i} \mathbf{y}_{i}^{\dagger}\right] \\
& =\mathbf{A} \mathbf{C} \mathbf{A}^{\dagger}+\sigma^{2} \mathbf{I}
\end{aligned}
$$

where $\mathbf{C}=E\left[\mathbf{c}_{i} \mathbf{c}_{i}^{\dagger}\right] \in \mathbb{C}^{2 K \times 2 K}$ is diagonal, $\mathbf{A}$ is defined in (7) and $\sigma^{2}$ is the noise variance. The correlation matrix can also be expressed in terms of its eigenvector decomposition

$$
\mathbf{R}=\mathbf{V D V}^{\dagger}
$$

where the columns of $\mathrm{V} \in \mathbb{C}^{N \times N}$ are the eigenvectors of $\mathbf{R}$, and $\mathbf{D}$ is a diagonal matrix of the corresponding eigenvalues $\left(\lambda_{n}\right)$. Furthermore

$$
\lambda_{n}= \begin{cases}d_{n}+\sigma^{2}, & \text { if } n \leq 2 K \\ \sigma^{2}, & \text { otherwise }\end{cases}
$$

where $d_{n}$ is the variance of the signal vectors along the $n$th eigenvector and we assume that $2 K<N$. Since the $2 K$ largest eigenvalues of $\mathbf{R}$ correspond to the signal subspace, $\mathbf{V}$ can be partitioned as $\mathbf{V}=\left[\begin{array}{ll}\mathbf{V}_{\mathcal{S}} & \mathbf{V}_{\mathcal{N}}\end{array}\right]$, where the columns of $\mathbf{V}_{\mathcal{S}}=\left[\begin{array}{lll}\mathbf{v}_{\mathcal{S}, 1} & \cdots & \mathbf{v}_{\mathcal{S}, 2 K}\end{array}\right] \in \mathbb{C}^{N \times 2 K}$ form a basis for the signal subspace $\mathcal{S}_{Y}$ and $\mathbf{V}_{\mathcal{N}}=\left[\begin{array}{llll}\mathbf{v}_{\mathcal{N}, 1} & \cdots & \mathbf{v}_{\mathcal{N}, N-2 K}\end{array}\right] \in$ $\mathbb{C}^{N \times N-2 K}$ spans the noise subspace $\mathcal{N}_{Y}$.

Since we would like to track slowly varying parameters, we form a moving average or Bartlett estimate of the correlation matrix based on the $J$ most recent observations

$$
\hat{\mathbf{R}}_{i}=\frac{1}{J} \sum_{j=i-J+1}^{i} \mathbf{y}_{j} \mathbf{y}_{j}^{\dagger} .
$$

It is well known [20] that the maximum likelihood estimate of the eigenvalues and associated eigenvectors of $\mathbf{R}$ is just the eigenvector decomposition of $\hat{\mathbf{R}}_{i}$. Thus, we perform an eigenvector decomposition of $\hat{\mathbf{R}}_{i}$, and select the eigenvectors corresponding to the $2 K$ largest eigenvalues as a basis for $\hat{\mathcal{S}}_{Y}$.

\section{Channel Estimation}

Consider the projection of a given user's signal vectors into the estimated noise subspace

$$
\left.\begin{array}{l}
\mathbf{e}_{k}^{r}=\left(\mathbf{u}_{k}^{r \dagger} \hat{\mathbf{V}}_{\mathcal{N}}\right)^{\top} \\
\mathbf{e}_{k}^{l}=\left(\mathbf{u}_{k}^{l \dagger} \hat{\mathbf{V}}_{\mathcal{N}}\right)^{\top}
\end{array}\right\} \in \mathbb{C}^{N-2 K} .
$$


Ideally, since the $\mathbf{u}_{k}$ 's lie completely within the signal subspace, the $\mathbf{e}_{k}$ 's should be identically zero. The channel estimate could then be formed by choosing a feasible $\mathrm{h}_{k}$ such that the corresponding signal vectors lie in the null space of $\hat{\mathbf{V}}_{\mathcal{N}}$. Furthermore, our assumptions on the invertibility of the map $G$ would guarantee this solution to be unique.

However, since we are dealing with an estimate and not the true noise subspace, the $\mathbf{e}_{k}$ 's are realizations of random vectors, and thus with probability one, $\mathbf{e}_{k}^{l}$ and $\mathbf{e}_{k}^{r}$ do not equal zero. Thus, we must introduce some measure of what is a "good" $\mathbf{e}_{k}$, so that we can choose $\mathbf{h}_{k}$ accordingly. We will explore two approaches to this problem. The first is a probabilistic approach apparently first proposed in [21] which exploits known statistical properties of the estimated subspaces to maximize the likelihood of the projections. The other is a geometric solution which seeks to minimize the distance (or $\ell_{2}$-norm) of the $\mathbf{e}_{k}$ 's. This is the well-known MUSIC algorithm [22], which is commonly used in array signal processing. Due to the absence of a displacement structure in our correlation matrix, ESPRIT techniques [18] are not applicable to our problem [23]. They would be applicable when our algorithms are extended to the multisensor case [23].

1) Probabilistic Approach: It has been shown [24] that the projections of the estimated noise subspace eigenvectors $\left\{\hat{\mathbf{v}}_{\mathcal{N}, i}\right\}$ onto the actual signal subspace $\mathbf{V}_{\mathcal{S}}$ are asymptotically jointly Gaussian with zero mean and covariance matrices given by

$$
\begin{aligned}
E\left[\left(\mathbf{V}_{\mathcal{S}} \mathbf{V}_{\mathcal{S}}^{\dagger} \hat{\mathbf{v}}_{\mathcal{N}, i}\right)\left(\mathbf{V}_{\mathcal{S}} \mathbf{V}_{\mathcal{S}}^{\dagger} \hat{\mathbf{v}}_{\mathcal{N}, j}\right)^{\dagger}\right]=\frac{1}{J} \mathbf{Q} \delta_{i, j} \\
E\left[\left(\mathbf{V}_{\mathcal{S}} \mathbf{V}_{\mathcal{S}}^{\dagger} \hat{\mathbf{v}}_{\mathcal{N}, i}\right)\left(\mathbf{V}_{\mathcal{S}} \mathbf{V}_{\mathcal{S}}^{\dagger} \hat{\mathbf{v}}_{\mathcal{N}, j}\right)^{\top}\right]=\mathbf{0}, \quad \text { for all } i, j
\end{aligned}
$$

where $\delta_{i, j}$ is the Kronecker delta function and

$$
\mathbf{Q}=\sigma^{2}\left[\sum_{k=1}^{2 K} \frac{\lambda_{k}}{\left(\sigma^{2}-\lambda_{k}\right)^{2}} \mathbf{v}_{\mathcal{S}, k} \mathbf{v}_{\mathcal{S}, k}^{\dagger}\right]
$$

Note, that in the above expressions we are dealing with the actual signal subspace, not the estimate. Thus

$$
\begin{aligned}
\mathbf{u}_{k}^{r \dagger} & =\mathbf{u}_{k}^{r \dagger} \mathbf{V}_{\mathcal{S}} \mathbf{V}_{\mathcal{S}}^{\dagger} \\
\mathbf{u}_{k}^{l \dagger} & =\mathbf{u}_{k}^{l \dagger} \mathbf{V}_{\mathcal{S}} \mathbf{V}_{\mathcal{S}}^{\dagger}
\end{aligned}
$$

and the projections into the estimated noise subspace can be rewritten as

$$
\begin{aligned}
\mathbf{e}_{k}^{r} & =\left(\mathbf{u}_{k}^{r \dagger} \mathbf{V}_{\mathcal{S}} \mathbf{V}_{\mathcal{S}}^{\dagger} \hat{\mathbf{V}}_{\mathcal{N}}\right)^{\top} \\
\mathbf{e}_{k}^{l} & =\left(\mathbf{u}_{k}^{l \dagger} \mathbf{V}_{\mathcal{S}} \mathbf{V}_{\mathcal{S}}^{\dagger} \hat{\mathbf{V}}_{\mathcal{N}}\right)^{\top} .
\end{aligned}
$$

Comparing this with (20), it is clear that asymptotically the $\mathbf{e}_{k}$ 's are also zero-mean, complex-valued, circular Gaussian random vectors. However, the projections are not statistically independent, thus we define a new $\mathbf{e}_{k}$ in order to arrive at a more tractable log-likelihood function. Clearly, if $\mathbf{u}_{k}^{r}$ and $\mathbf{u}_{k}^{l}$ both lie in the signal subspace, then their sum $\mathbf{u}_{k}=\mathbf{u}_{k}^{r}+\mathbf{u}_{k}^{l}$ must also be contained in $\mathbf{V}_{\mathcal{S}}$. We now consider the projection of $\mathbf{u}_{k}$ into the estimated noise subspace

$$
\tilde{\mathbf{e}}_{k}=\left(\mathbf{u}_{k}^{\dagger} \hat{\mathbf{V}}_{\mathcal{N}}\right)^{\top}
$$

This too is a Gaussian random vector and thus has probability density function

$$
p_{\tilde{\mathbf{e}}}\left(\tilde{\mathbf{e}}_{k}\right)=\frac{1}{\operatorname{det}[\pi \mathbf{K}]} \exp \left\{-\tilde{\mathbf{e}}_{k}^{\dagger} \mathbf{K}^{-1} \tilde{\mathbf{e}}_{k}\right\} .
$$

From (20), we see that the covariance matrix $\mathbf{K}$ is a scalar multiple of the identity given by

$$
\mathbf{K}=\frac{1}{J} \mathbf{u}_{k}^{\dagger} \mathbf{Q} \mathbf{u}_{k} \mathbf{I} .
$$

Therefore, within an additive constant, the log-likelihood function of $\tilde{\mathbf{e}}_{k}$ is

$$
\begin{aligned}
\Lambda\left(\tilde{\mathbf{e}}_{k}\right)= & -(N-2 K) \ln \left(\mathbf{u}_{k}^{\dagger} \mathbf{Q} \mathbf{u}_{k}\right)-J \frac{\tilde{\mathbf{e}}_{k}^{\dagger} \tilde{\mathbf{e}}_{k}}{\mathbf{u}_{k}^{\dagger} \mathbf{Q} \mathbf{u}_{k}} \\
= & -(N-2 K) \ln \left(\mathbf{u}_{k}^{\dagger} \mathbf{Q} \mathbf{u}_{k}\right) \\
& -J \frac{\mathbf{u}_{k}^{\dagger} \mathbf{V}_{\mathcal{N}} \mathbf{V}_{\mathcal{N}}^{\dagger} \mathbf{u}_{k}}{\mathbf{u}_{k}^{\dagger} \mathbf{Q u} \mathbf{u}_{k}} .
\end{aligned}
$$

Of course, the exact $\mathbf{V}_{\mathcal{N}}$ and $\mathbf{Q}$ are unknown, but we may replace them with their estimates.

Unfortunately, maximizing this likelihood function is prohibitively complex for a general multipath channel, so we will consider only a single propagation path. In this case, the vector $\mathbf{u}_{k}$ is a function of only one unknown parameter: the delay $\tau_{k}$. To form the timing estimate, we must solve

$$
\hat{\tau}_{k}=\underset{\tau_{k} \in[0, T)}{\arg \max } \Lambda\left(\mathbf{u}_{k}\right) .
$$

Ideally, we would like to differentiate the log-likelihood function with respect to $\tau$. However, the desired user's delay lies within an uncertainty region, $\tau_{k} \in[0, T]$, and $\mathbf{u}_{k}(\tau)$ is only piecewise continuous on this interval. To counter these problems, we divide the uncertainty region into $N$ cells of width $T_{c}$ and consider a single cell, $C_{\nu} \equiv\left[\nu T_{c},(\nu+1) T_{c}\right)$. We again define $\nu \in\{0, \cdots, N-1\}$ and $\gamma \in[0,1)$ such that $\left(\tau / T_{c}\right) \bmod N=\nu+\gamma$, and for $\tau \in C_{\nu}$ the desired user's signal vector becomes

$$
\mathbf{u}_{k}(\tau)=(1-\gamma) \mathbf{u}_{k}(\nu)+\gamma \mathbf{u}_{k}(\nu+1)
$$

and

$$
\begin{aligned}
\frac{d}{d \tau} \mathbf{u}_{k}(\tau) & =\mathbf{u}_{k}(\nu+1)-\mathbf{u}_{k}(\nu) \\
& =\text { a constant. }
\end{aligned}
$$

Thus, within a given cell, we can differentiate the loglikelihood function and solve for the maximum in closed form. We then choose whichever of the $N$ solutions yields the largest value for (27).

To determine the stationary points of the log-likelihood function, we first compute the following $4 N$ scalars

$$
\begin{aligned}
& x_{1}(\nu)=\mathbf{u}_{k}^{\dagger}(\nu) \mathbf{Q} \mathbf{u}_{k}(\nu) \\
& x_{2}(\nu)=\mathbf{u}_{k}^{\dagger}(\nu) \mathbf{Q} \mathbf{u}_{k}(\nu+1) \\
& x_{3}(\nu)=\mathbf{u}_{k}^{\dagger}(\nu) \mathbf{V}_{\mathcal{N}} \mathbf{V}_{\mathcal{N}}^{\dagger} \mathbf{u}_{k}(\nu) \\
& x_{4}(\nu)=\mathbf{u}_{k}^{\dagger}(\nu) \mathbf{V}_{\mathcal{N}} \mathbf{V}_{\mathcal{N}}^{\dagger} \mathbf{u}_{k}(\nu+1) .
\end{aligned}
$$




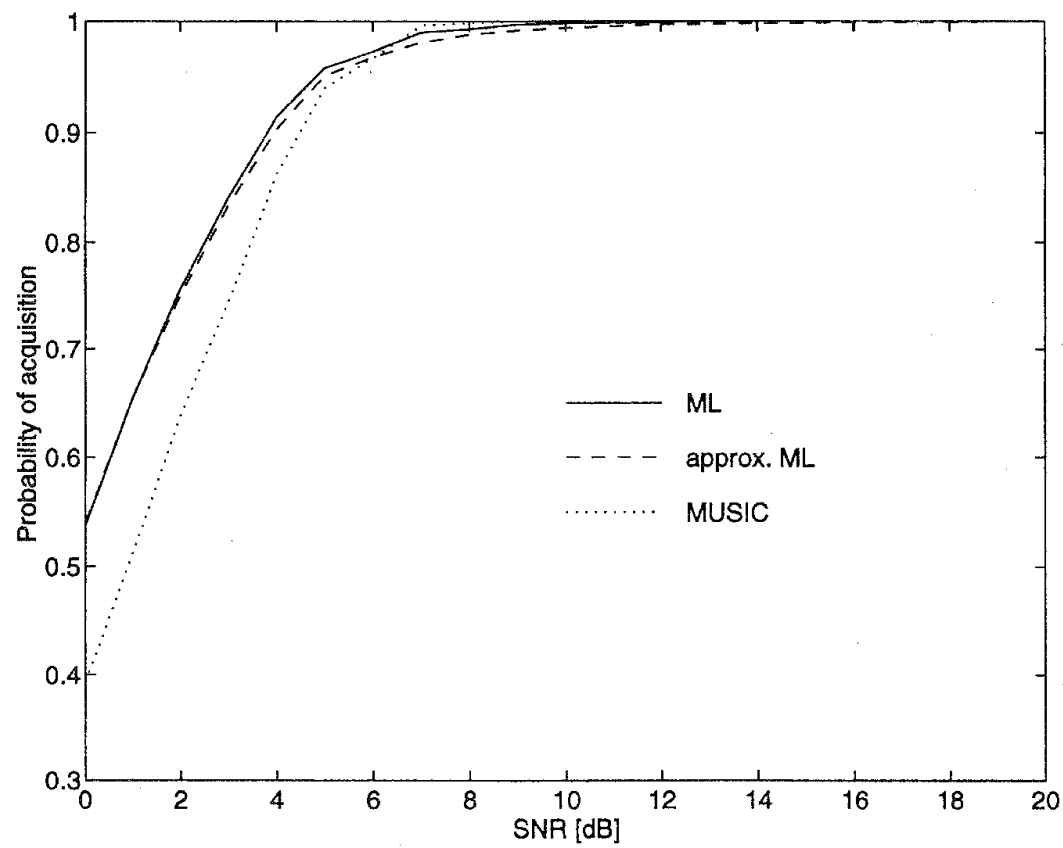

Fig. 2. Probability of acquisition for the maximum likelihood (ML) estimator, the approximate ML, and the MUSIC algorithm $[K=5, N=31, J=200$, MAI $=20 \mathrm{~dB}]$.

For each cell, $C_{\nu} \equiv\left[\nu T_{c},(\nu+1) T_{c}\right)$, we calculate

$$
\begin{aligned}
& x_{1}=x_{1}(\nu)-2 x_{2}(\nu)+x_{1}(\nu+1) \\
& \dot{x}_{1}=x_{2}(\nu)-x_{1}(\nu+1) \\
& \ddot{x}_{1}=x_{1}(\nu+1) \\
& x_{3}=x_{3}(\nu)-2 x_{4}(\nu)+x_{3}(\nu+1) \\
& \dot{x}_{3}=x_{4}(\nu)-x_{3}(\nu+1) \\
& \ddot{x}_{3}=x_{3}(\nu+1)
\end{aligned}
$$

and from these compute

$$
\begin{aligned}
a= & (1-J) x_{1} x_{3}-(N-2 K) u^{2} \\
b= & (2-J) x_{1} \dot{x}_{3}+(1-2 J) \dot{x}_{1} x_{3} \\
& -3(N-2 K) u \dot{x}_{1} \\
c= & 2(1-J) \dot{x}_{1} \dot{x}_{3}+\ddot{x}_{3} x_{1}-J \ddot{x}_{1} x_{3} \\
& \quad-(N-2 K)\left(2 \dot{x}_{1}^{2}+\ddot{x}_{1} x_{1}\right) \\
d= & \ddot{x}_{3} \dot{x}_{1}-J \ddot{x}_{1} \dot{x}_{3}-(N-2 K) \ddot{x}_{1} \dot{x}_{1} .
\end{aligned}
$$

Within the cell $C_{\nu}$, there are stationary points at $\tau=(\nu+$ $\left.\gamma^{*}\right) T_{c}$, where

$$
\gamma^{*} \in\left\{\gamma: a \gamma^{3}+b \gamma^{2}+c \gamma+d=0 \text { and } \gamma \in[0,1)\right\}
$$

Since $C_{\nu}$ is a half-open interval, it need not contain a local maximum at all. However, if it does, that local maximum must occur at either $\gamma=0$ or at one of the stationary points. We compute the likelihood function at each of these points and select the one with the greatest value.

Under certain conditions, it may be possible to simplify this algorithm. Note that maximizing the log-likelihood function
(27) is equivalent to maximizing

$$
\begin{aligned}
\Lambda\left(\tilde{\mathbf{e}}_{k}\right)= & -\frac{N-2 K}{J} \ln \left(\mathbf{u}_{k}^{\dagger} \mathbf{Q} \mathbf{u}_{k}\right) \\
& -\frac{\mathbf{u}_{k}^{\dagger} \mathbf{V}_{\mathcal{N}} \mathbf{V}_{\mathcal{N}}^{\dagger} \mathbf{u}_{k}}{\mathbf{u}_{k}^{\dagger} \mathbf{Q} \mathbf{u}_{k}}
\end{aligned}
$$

As $J \rightarrow \infty$, the leading term goes to zero; thus, for large observation windows, we can use the following approximation:

$$
\Lambda\left(\tilde{\mathbf{e}}_{k}\right) \approx-\frac{\mathbf{u}_{k}^{\dagger} \mathbf{V}_{\mathcal{N}} \mathbf{V}_{\mathcal{N}}^{\dagger} \mathbf{u}_{k}}{\mathbf{u}_{k}^{\dagger} \mathbf{Q} \mathbf{u}_{k}}
$$

This yields a much simpler expression for the stationary points. For each cell $C_{\nu}$, we define

$$
\begin{aligned}
\begin{array}{c}
a= \\
=
\end{array} & \left.x_{3}(\nu+1)-x_{3}(\nu)\right] \operatorname{Re}\left\{x_{2}(\nu)\right\} \\
& -\left[x_{1}(\nu+1)-x_{1}(\nu)\right] \operatorname{Re}\left\{x_{4}(\nu)\right\} \\
& +x_{3}(\nu) x_{1}(\nu+1)-x_{3}(\nu+1) x_{1}(\nu) \\
b= & 2 x_{3}(\nu) \operatorname{Re}\left\{x_{2}(\nu)\right\}-2 x_{1}(\nu) \operatorname{Re}\left\{x_{4}(\nu)\right\} \\
& -x_{3}(\nu) x_{1}(\nu+1)+x_{3}(\nu+1) x_{1}(\nu) \\
c= & x_{1}(\nu) \operatorname{Re}\left\{x_{4}(\nu)\right\}-x_{3}(\nu) \operatorname{Re}\left\{x_{2}(\nu)\right\}
\end{aligned}
$$

and the stationary points are located at

$$
\gamma^{*} \in\left\{\gamma: a \gamma^{2}+b \gamma+c=0 \text { and } \gamma \in[0,1)\right\} .
$$

It is interesting to note that the MUSIC algorithm is equivalent to (36) when one only maximizes the numerator and ignores the denominator, i.e., one assumes $\mathbf{u}_{k}^{\dagger} \mathbf{Q} \mathbf{u}_{k}$ is equal to one in (35) or (36). This yields an even simpler approximation for the log-likelihood function

$$
\Lambda\left(\tilde{\mathbf{e}}_{k}\right) \approx-\mathbf{u}_{k}^{\dagger} \mathbf{V}_{\mathcal{N}} \mathbf{V}_{\mathcal{N}}^{\dagger} \mathbf{u}_{k}
$$




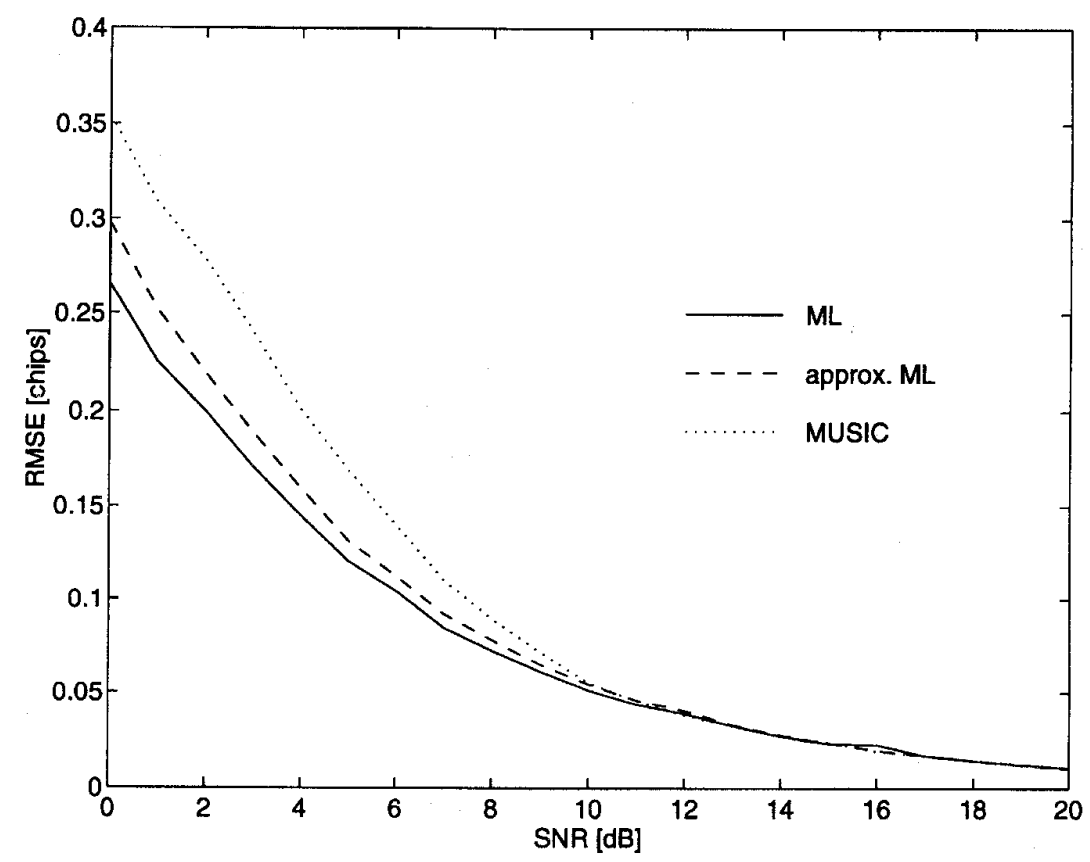

Fig. 3. Root mean squared error (RMSE) of the delay estimate in chips for the maximum likelihood (ML) estimator, the approximate ML, and the MUSIC algorithm $[K=5, N=31, J=200$, MAI $=20 \mathrm{~dB}]$.

which allows a linear solution for the stationary points

$$
\gamma^{*}=\frac{x_{3}(\nu)-\operatorname{Re}\left\{x_{4}(\nu)\right\}}{x_{3}(\nu)-2 \operatorname{Re}\left\{x_{4}(\nu)\right\}+x_{3}(\nu+1)} .
$$

2) Geometric Approach: From the preceding subsection, it is clear that we must define a less complicated cost function if we are to consider general multipath channels. Thus, we look for an alternative to our stochastic model and consider a nonprobabilistic method. We would like the projections of the signal vectors into the noise subspace to be zero, however as mentioned earlier, we can never achieve this in practice. Intuitively, however, we have the sense that the "smaller" the projections are, the "better" our estimate is. To form our channel estimate, we propose to minimize the Euclidean distance or $\ell_{2}$ norm of the projections. Thus, our channel estimate $\hat{\mathbf{h}}_{k}$ is the solution to the quadratic program

$$
\hat{\mathbf{h}}_{k}=\underset{\mathbf{h} \in \mathcal{H}}{\arg \min }\left\|\mathbf{u}_{k}^{r \dagger} \hat{\mathbf{V}}_{\mathcal{N}}\right\|^{2}+\left\|\mathbf{u}_{k}^{l \dagger} \hat{\mathbf{V}}_{\mathcal{N}}\right\|^{2}
$$

where the constraint set $\mathcal{H}$ is the set of all feasible impulse responses as determined by some a priori channel model. Using the matrix representation for the signal vectors given by (13), the program becomes

$$
\hat{\mathbf{h}}_{k}=\underset{h \in \mathcal{H}}{\arg \min } \mathbf{h}^{\dagger}\left[\mathbf{U}_{k}^{l \dagger} \hat{\mathbf{V}}_{\mathcal{N}} \hat{\mathbf{V}}_{\mathcal{N}}^{\dagger} \mathbf{U}_{k}^{l}+\mathbf{U}_{k}^{r \dagger} \hat{\mathbf{V}}_{\mathcal{N}} \hat{\mathbf{V}}_{\mathcal{N}}^{\dagger} \mathbf{U}_{k}^{r}\right] \mathbf{h} .
$$

Depending on the constraint set, this optimization problem may be quite difficult to solve, so we propose a two-step approximate solution. Since a given user's transmitted amplitude and phase are unknown, we can only determine $\hat{h}_{k}$ to within a complex multiplicative constant, so without loss of generality we can introduce the simple constraint $\|\mathbf{h}\|=1$. If we define $\mathbf{M}=\mathbf{U}_{k}^{l \dagger} \hat{\mathbf{V}}_{\mathcal{N}} \hat{\mathbf{V}}_{\mathcal{N}}^{\dagger} \mathbf{U}_{k}^{l}+\mathbf{U}_{k}^{r \dagger} \hat{\mathbf{V}}_{\mathcal{N}} \hat{\mathbf{V}}_{\mathcal{N}}^{\dagger} \mathbf{U}_{k}^{r}$, then the quadratic program reduces to

$$
\hat{\mathbf{h}}_{k}=\underset{\|\mathbf{h}\|=1}{\arg \min } \mathbf{h}^{\dagger} \mathbf{M h}
$$

In the absence of noise, $M$ will not have full rank, and the true $\mathbf{h}_{k}$ will lie in the null space of $\mathbf{M}$. Thus, our conditions for identifiability are equivalent to assuming that this null space has rank one, in which case there is a unique solution to the optimization problem. However, in the presence of noise, $\mathbf{M}$ will have full rank, and the solution is simply the eigenvector corresponding to the smallest eigenvalue of $\mathbf{M}$.

In many cases, we may have no a priori information about the channel, and thus $\mathcal{H}=\mathbb{C}^{N}$. In this case, our estimate $\hat{\mathbf{h}}_{k}$ is trivially contained in the set of feasible impulse responses. However, many techniques used to combat multipath (e.g., RAKE correlators and matched filter banks) assume that the channel impulse response can be modeled as a finite number of distinct propagation paths. Due to noise, $\hat{\mathbf{h}}_{k}$ will generally not satisfy this constraint, so we now perform a least-squares fit to our parametric channel model to extract the strongest paths.

It is important to remember that a single path will manifest itself in two adjacent values of the composite channel impulse response. We now perform a least-squares fit of a single path to each pair of adjacent coefficients,

$$
\begin{aligned}
{\left[\alpha_{\nu}, \gamma_{\nu}\right]=} & \underset{\alpha \in \mathbb{C}, \gamma \in[0,1)}{\arg \min }\left|\hat{h}_{k, \nu}-(1-\gamma) \alpha\right|^{2} \\
& +\left|\hat{h}_{k, \nu+1}-\gamma \alpha\right|^{2}
\end{aligned}
$$




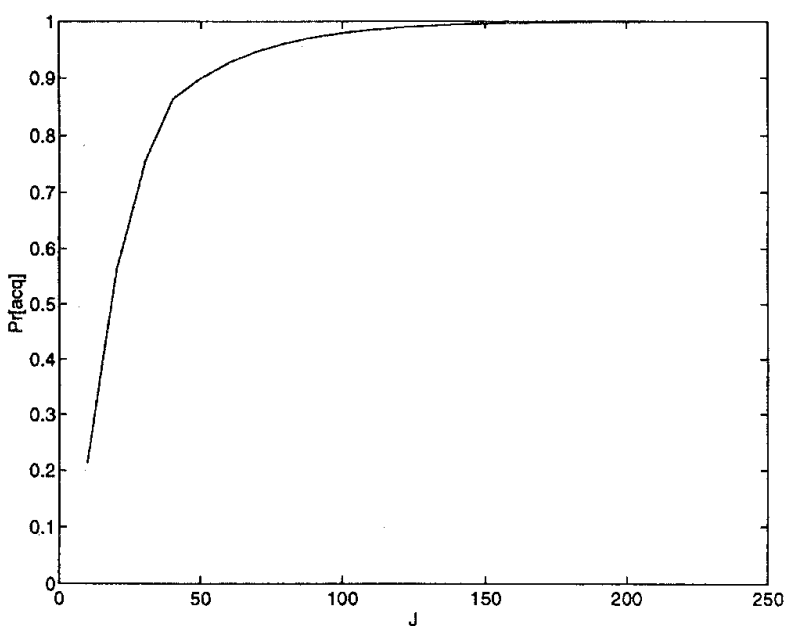

(a)

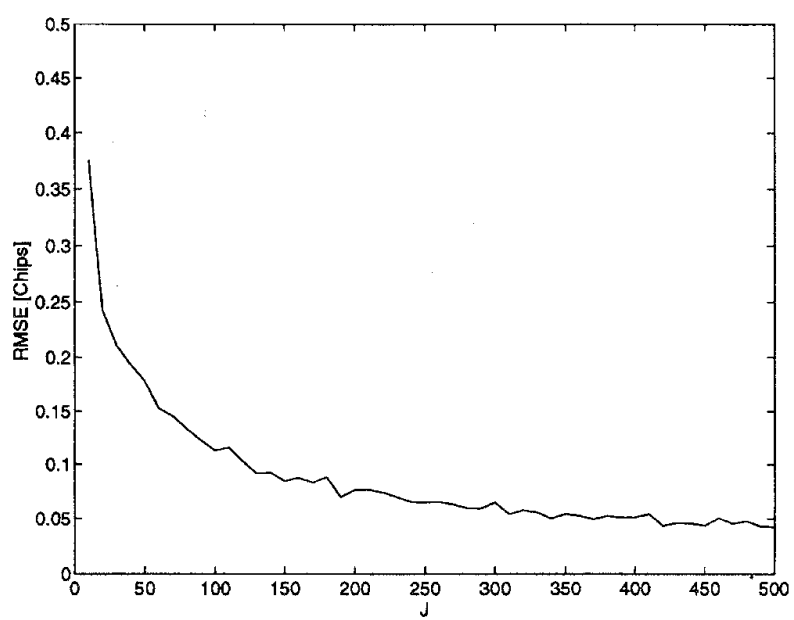

(b)

Fig. 4. (a) Probability of acquisition and (b) root mean squared error (RMSE) of timing estimate in chips of the subspace-based maximum likelihood estimator for varying window size $[N=31, \mathrm{SNR}=8 \mathrm{~dB}, K=5$, MAl $=20 \mathrm{~dB}]$.

where $\hat{h}_{k, \nu}$ is the $\nu$ th element of $\hat{\mathbf{h}}_{k}$. The solution to the least-squares problem is given by

$$
\begin{aligned}
\gamma_{\nu} & =\frac{1}{2}+\beta \pm \sqrt{\beta^{2}+\frac{1}{2}} \\
\alpha_{\nu} & =\frac{\left(1-\gamma_{\nu}\right) \hat{h}_{k, \nu}+\gamma_{\nu} \hat{h}_{k, \nu+1}}{\left(1-\gamma_{\nu}\right)^{2}+\gamma_{\nu}^{2}}
\end{aligned}
$$

where

$$
\beta=\frac{\operatorname{Re}\left\{\overline{\hat{h}}_{k, \nu} \hat{h}_{k, \nu+1}\right\}}{\left|\hat{h}_{k, \nu}\right|^{2}-\left|\hat{h}_{k, \nu+1}\right|^{2}} .
$$

Recall that we need to only consider $\gamma_{\nu} \in[0,1)$. All that remains is to search for the strongest path

$$
\hat{\nu}=\underset{\nu \in\{0,1, \cdots, N-1\}}{\arg \max }\left|\alpha_{\nu}\right|
$$

and then compute the desired channel parameters

$$
\hat{\tau}=\left(\hat{\nu}+\gamma_{\hat{\nu}}\right) T_{c}
$$

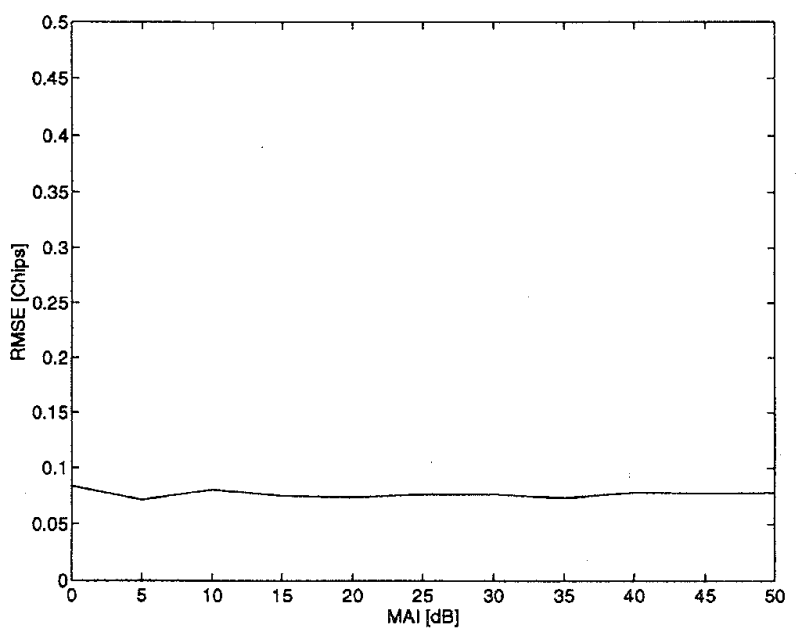

(a)

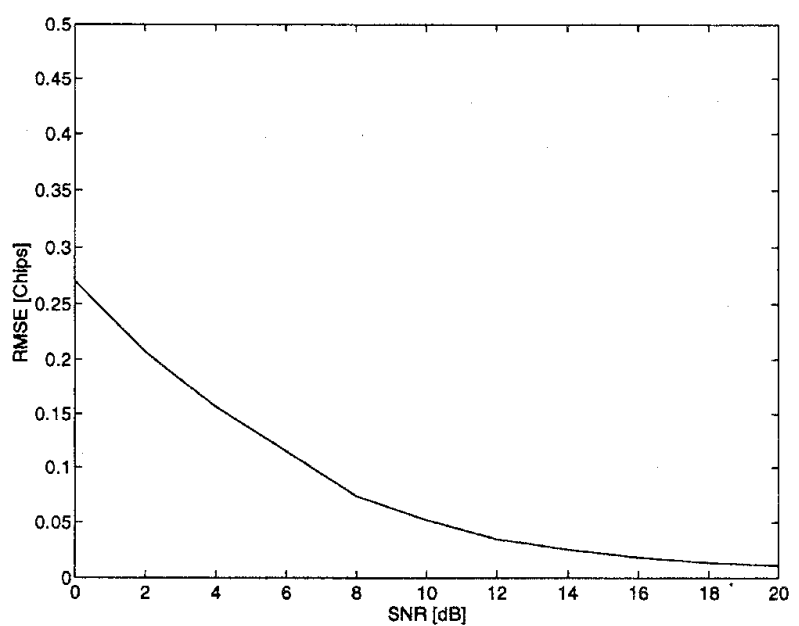

(b)

Fig. 5. RMSE of the subspace-based maximum likelihood estimator for varying (a) MAI level $[J=200, N=31, \mathrm{SNR}=8 \mathrm{~dB}, K=5]$ and (b) SNR values $[J=200, N=31, K=5, \mathrm{MAI}=20 \mathrm{~dB}]$.

and

$$
\hat{\alpha}=\alpha_{\hat{\nu}}
$$

The estimated path is subtracted from $\hat{\mathrm{h}}_{k}$, and the process is repeated to find the next strongest path, etc., until either a specified number of paths have been identified or $|\hat{\alpha}|$ falls below some predetermined significance level. In addition, subsequent searches can be restricted to a range of $\nu$ consistent with the assumed multipath spread.

\section{Estimation of Received Amplitude}

In the presentation of these subspace-based methods, the amplitude and phase have been lumped into arbitrary complex coefficients and thus obscured. Since second order statistics contain no phase information, we clearly can not recover phase from the sample correlation matrix. However, we now consider a subspace-based method for estimating the received amplitude. 


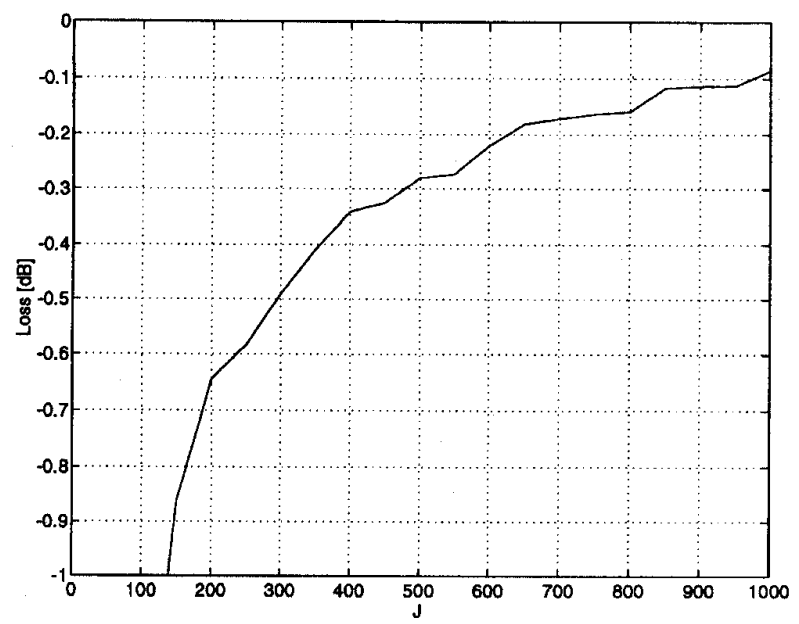

(a)

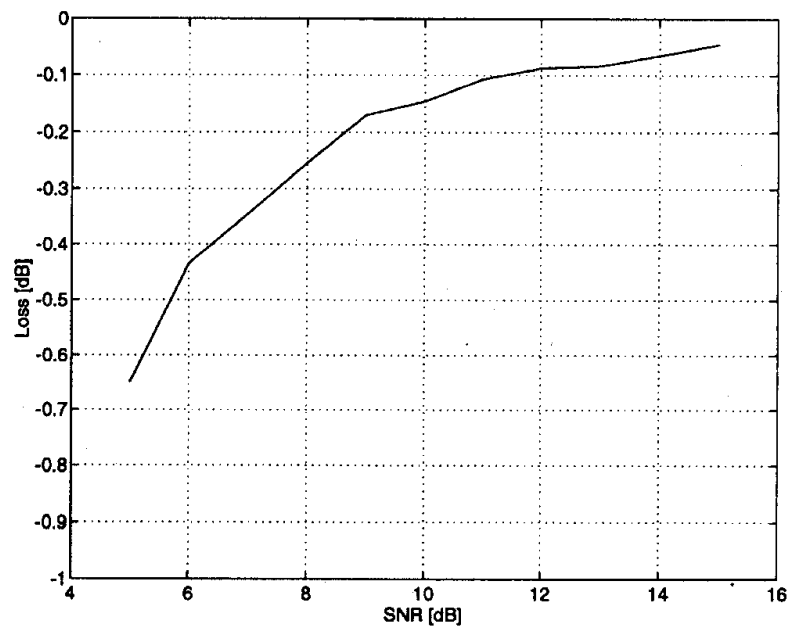

(b)

Fig. 6. (a) Loss in SNR at the output of a RAKE correlator using estimated parameters versus one with perfect knowledge for varying observation window $[N=31, K=5$, SNR $=8 \mathrm{~dB}, \mathrm{MAI}=20 \mathrm{~dB}, 3$ paths $]$ and (b) loss for varying $\mathrm{SNR}[J=500, N=31, K=5, \mathrm{MAI}=20 \mathrm{~dB}, 3$ paths $]$.

Since we assume that the channel attenuation parameters vary slowly and we are using PSK for all data and spreading modulation, the users' signals have constant modulus, i.e., $\left|c_{k}^{(i)}\right|=\left|c_{k}\right|=$ a constant. Thus, estimating the received amplitude is equivalent to estimating $\left|c_{k}\right|$. Recall that the correlation matrix has the form

$$
\begin{aligned}
\mathbf{R} & =\mathbf{A C A}^{\dagger}+\sigma^{2} \mathbf{I} \\
& \equiv \hat{\mathbf{R}}_{\mathcal{S}}+\sigma^{2} \mathbf{I}
\end{aligned}
$$

We now extract the signal correlation matrix $\hat{\mathbf{R}}_{\mathcal{S}}$ from the total correlation matrix $\hat{\mathbf{R}}$ by finding the matrix of this form that best fits the sample correlation matrix in a two-norm sense, i.e.,

$$
\left[\begin{array}{ll}
\hat{\mathbf{R}}_{\mathcal{S}} & \hat{\sigma}^{2}
\end{array}\right]=\underset{\mathbf{R}_{\mathcal{S}}, \sigma^{2}}{\arg \min }\left\|\hat{\mathbf{R}}-\mathbf{R}_{\mathcal{S}}-\sigma^{2} \mathbf{I}\right\|_{2}
$$

subject to $\sigma^{2} \in \mathbb{R}^{+}$and $\mathbf{R}_{\mathcal{S}} \in \mathbb{C}^{N \times N}$ a positive, semidefinite matrix of rank $2 K$. This has the solution

$$
\begin{aligned}
\hat{\sigma}^{2} & =\frac{1}{2}\left(\lambda_{2 K+1}+\lambda_{N}\right) \\
\hat{\mathbf{R}}_{\mathcal{S}} & =\hat{\mathbf{V}}_{\mathcal{S}} \tilde{\mathbf{D}}_{\mathcal{S}}^{\dagger}
\end{aligned}
$$

where $\tilde{\mathbf{D}}=\operatorname{diag}\left(\lambda_{1}-\hat{\sigma}^{2}, \cdots, \lambda_{2 K}-\hat{\sigma}^{2}\right)$. Since we have already estimated the channel parameters, we have in effect also formed estimates for the signal vectors $\hat{\mathbf{u}}_{k}^{r}$ and $\hat{\mathbf{u}}_{k}^{l}$. We define the signal matrix for a single user as

$$
\mathbf{A}_{k}=\left[\begin{array}{ll}
\hat{\mathbf{u}}_{k}^{r} & \hat{\mathbf{u}}_{k}^{l}
\end{array}\right] \in \mathbb{C}^{N \times 2} .
$$

Ideally, we would like to choose $\left|\hat{c}_{k}\right|$ such that $\hat{\mathbf{R}}_{\mathcal{S}}-$ $\left|\hat{c}_{k}\right|^{2} \mathbf{A}_{k} \mathbf{A}_{k}^{\dagger}$ has rank $2(K-1)$ or equivalently

$$
\mathbf{I}-\left|\hat{c}_{k}\right|^{2} \mathbf{A}_{k}^{\dagger} \hat{\mathbf{R}}_{\mathcal{S}}^{-1} \mathbf{A}_{k}=\mathbf{0} .
$$

Of course, due to errors in our estimate of the signal correlation matrix, this will generally be impossible, so we instead seek the best fit in a two-norm sense

$$
\left|\hat{c}_{k}\right|=\underset{c_{k}}{\arg \min }\left\|\mathbf{I}-\left|\hat{c}_{k}\right|^{2} \mathbf{A}_{k}^{\dagger} \hat{\mathbf{R}}_{\mathcal{S}}^{-1} \mathbf{A}_{k}\right\|_{2}
$$

This has the solution

$$
\left|\hat{c}_{k}\right|=\left(\frac{\hat{\mathbf{u}}_{k}^{r \dagger} \hat{\mathbf{R}}_{\mathcal{S}}^{-1} \hat{\mathbf{u}}_{k}^{r}+\hat{\mathbf{u}}_{k}^{l \dagger} \hat{\mathbf{R}}_{\mathcal{S}}^{-1} \hat{\mathbf{u}}_{k}^{l}}{2}\right)^{-(1 / 2)}
$$

Note from (51) that the inverse of $\hat{\mathbf{R}}_{\mathcal{S}}$ is easily calculated, and thus the amplitude estimates require only minimal computation.

\section{NuMERICAL RESULTS}

We conducted simulations of the proposed algorithms for five users with length 31 Gold codes. A single desired user was acquired and tracked in the presence of strong multiple access interference (MAI). The power ratio between each of the four interfering users and the desired user is designated the MAI level.

We first compared the true log-likelihood estimate (27) with the large observation window approximation (36) and the MUSIC algorithm (39). This was done for a window size of 200 symbols and with a varying SNR. Fig. 2 shows the probability of acquisition for each method, where acquisition is defined as $\left|\tau_{k}-\hat{\tau}_{k}\right|<\frac{1}{2} T_{c}$. Using the approximate loglikelihood function resulted in almost no drop in performance. Furthermore, when the SNR was poor, both probabilistic approaches considerably outperformed the MUSIC algorithm. In Fig. 3, we compare the RMSE of the delay estimate once acquisition has occurred, i.e., after processing enough symbols to reach within half of one chip. The approximate log-likelihood function experiences a slight increase in error at low SNR, but again both probabilistic methods do better than MUSIC. It is interesting to note that for sufficiently high 


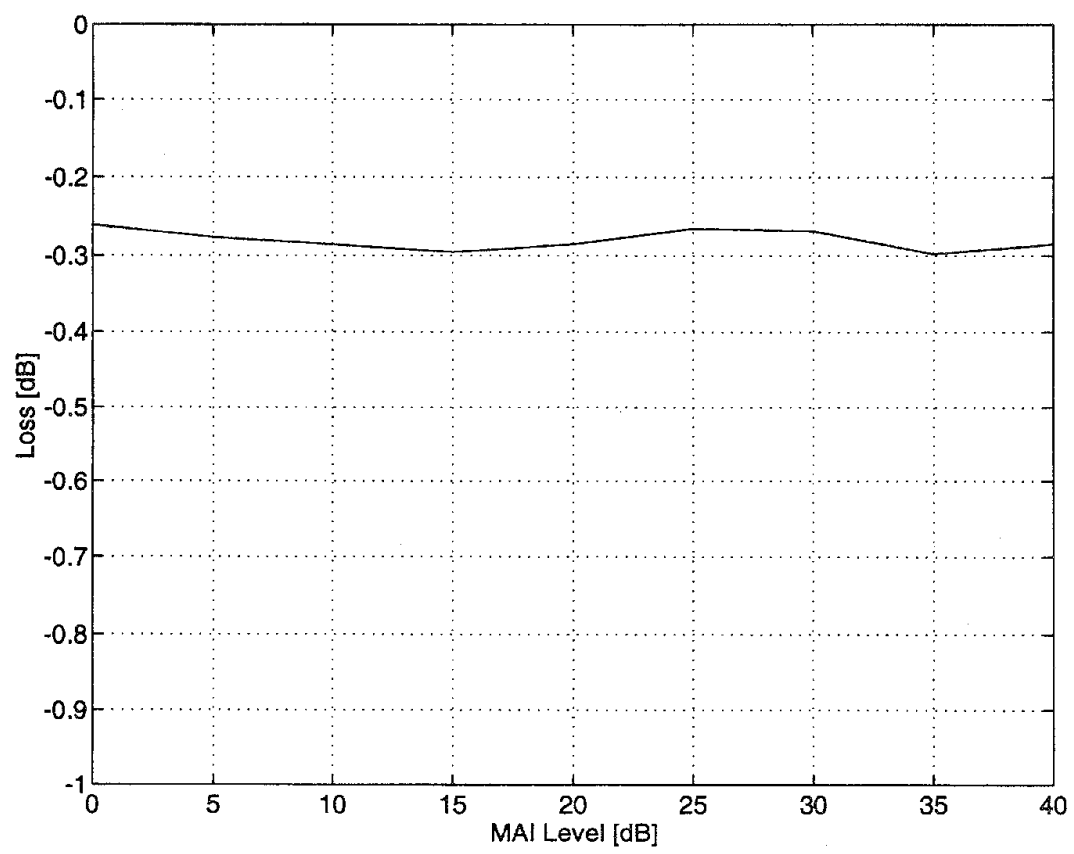

Fig. 7. Loss in SNR at the output of a RAKE correlator using estimated parameters versus one with perfect knowledge for varying MAI level $[J=500, N=31, \mathrm{SNR}=8 \mathrm{~dB}, K=5,3$ paths $]$.

SNR $(>10 \mathrm{~dB})$, there is virtually no difference in performance among the three methods.

We next take a more in depth look at the performance of the full maximum likelihood estimator. Fig. 4(a) demonstrates the algorithm's ability to acquire a desired user's delay even with large MAI. In Fig. 4(b), we examine the relationship between the RMSE of the timing estimate and the window size. Tracking errors on the order of 0.1 chips can be achieved with windows of less than 200 bits, indicating that the algorithm can be used for tracking of slowly time-varying parameters. Fig. 5(a) and (b) illustrate the near-far resistance of the algorithm. As expected, the estimate is influenced by the SNR, but even large changes in the MAI level have no effect on accuracy.

To evaluate the geometric approach we simulated a channel with three propagation paths. The propagation delays were generated as uniform random variables with $\tau_{1} \sim U[0, T)$ and $\tau_{2}, \tau_{3} \sim U\left[0, \frac{1}{2} T\right)+\tau_{1}$. The attenuation weights were complex Gaussian random variables with zero mean and equal variance.

Since a large number of interdependent parameters are being estimated, it is not very revealing to look at the estimation error for each parameter individually. Instead the estimated channel parameters are passed to a RAKE correlator [9] which is used to collect the signal energy from all the propagation paths. For a performance metric, we then calculated the loss in SNR at the output of a RAKE correlator using the estimated parameters as compared to one with perfect knowledge of the channel.

Fig. 6(a) shows the resulting loss in SNR as a function of the observation window length $J$. When choosing $J$, there is a natural trade-off between estimation error and tracking speed. In Fig. 6(b), we see the effect of varying SNR. As expected, the algorithm is influenced by the SNR. However, a given performance level can always be maintained by adjusting the window length. Thus, a poor SNR will only degrade the capability to track time varying parameters. In Fig. 7, we confirm that the algorithm is indeed near-far resistant, since even large changes in the MAI level have no effect on performance.

To gain some insight into how the proposed estimator effects the probability of error, we simulated a decorrelating receiver [25] using a bank of RAKE correlators matched to each user. In Fig. 8, we compare the probability of error when using the estimated parameters to a receiver using the true parameter values. We have also shown the probability of error for a decorrelating receiver using the standard matched filter front-end with perfect tracking of a single propagation path. The RAKE receiver using the estimated values performs almost identically to the receiver with perfect knowledge of the channel parameters, and both considerably outperform the receiver which neglects the multipath.

\section{CONCluston}

Since the signal subspace is determined solely by the users' spreading waveforms and not their relative amplitudes, subspace-based methods are both near-far resistant and wellsuited for fading channels. It is also interesting to note, that when calculating the estimate for a given user, no knowledge of the other users' spreading waveforms is necessary. Thus, the algorithm can be used for both multiuser or single user estimation. Although we limited our work to channels with multipath spreads of less than half the symbol period, longer delays could easily be accommodated by increasing the length of the observation vectors. 


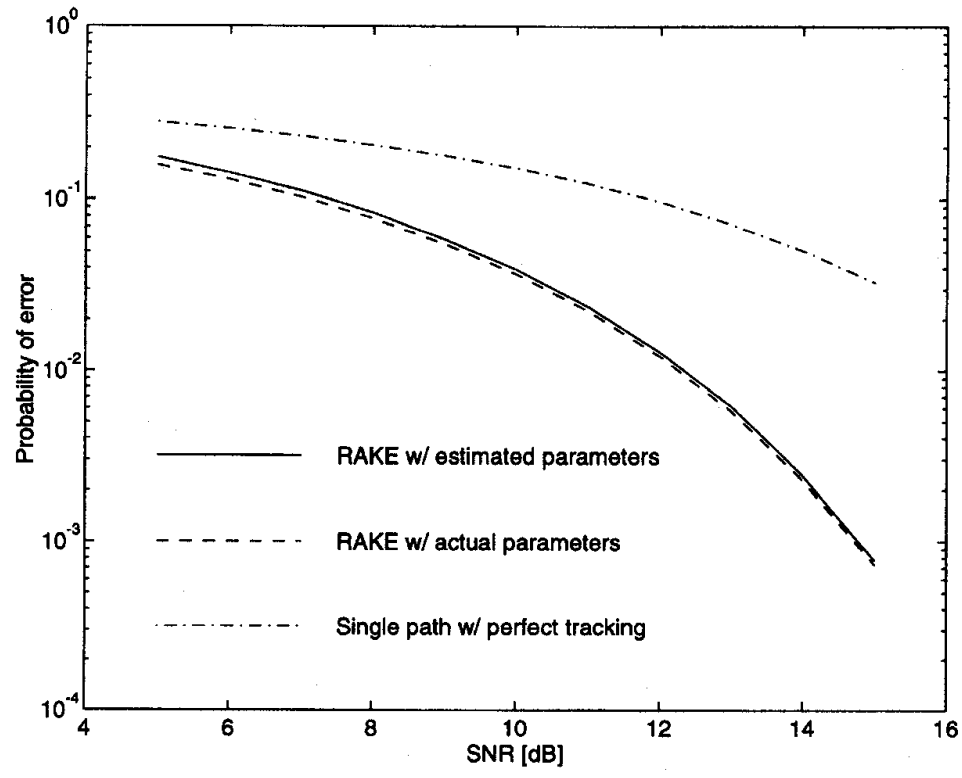

Fig. 8. Probability of error for a decorrelating receiver using RAKE correlators with actual parameter values; RAKE correlators with estimated parameter values; and a correlator matched to a single path $[J=500, N=31, K=5, \mathrm{MAI}=20 \mathrm{~dB}, 3$ paths $]$.

Furthermore, the estimate is formed solely from secondorder statistics, which are independent of the data modulated on the waveform. Thus, unlike other methods, no preamble or decision feedback is necessary. This makes the algorithm very stable during tracking, since it is able to quickly reacquire the desired user if it loses lock.

Perhaps the biggest drawback to subspace-based methods is the $O\left(N^{3}\right)$ computational complexity of performing an eigenvector decomposition. However, note that in the geometric approach, we don't need the actual eigenvector decomposition. All we really need is a set of orthonormal basis vectors for the noise subspace. A great deal of research has been done on reduced complexity subspace tracking [26], [27], including an $O(N K)$ algorithm based on the recursive least squares problem [28]. These techniques to reduce the computational complexity of the algorithm suggest that the approach may also find application, with reasonable hardware, in tracking slowly varying channel parameters in mobile systems.

\section{REFERENCES}

[1] R. L. Pickholtz, D. L. Schilling, and L. B. Milstein, "Theory of spreadspectrum communications-A tutorial," IEEE Trans. Commun., vol. COM-30, pp. 904-912, May 1982.

[2] T. K. Moon, R. T. Short, and C. K. Rushforth, "Average acquisition time for SSMA channels," in Proc. IEEE Military Commun. Conf., 1991, vol. 3, pp. 1042-1046.

[3] S. Verdú, "Minimum probability of error for asynchronous Gaussian multiple-access channels," IEEE Trans. Inform. Theory, vol. IT-32, pp. 85-96, Jan. 1986.

[4] R. Lupas and S. Verdú, "Linear multiuser detectors for synchronous code-division multiple-access channels," IEEE Trans. Inform. Theory, vol. IT-35, pp. 123-136, Jan. 1989.

[5] M. K. Varanasi and B. Aazhang, "Multistage detection in asynchronous code-division multiple-access communications," IEEE Trans. Commun., vol. 38, pp. 509-519, Apr. 1990.

[6] U. Madhow and M. L. Honig, "MMSE interference suppression for direct-sequence spread-spectrum CDMA," IEEE Trans. Commun., vol. 42, pp. $3178-3188$, Dec. 1994.
[7] A. A. M. Saleh and R. A. Valenzuela, "A statistical model for indoor multipath propagation," IEEE J. Select. Areas Commun., vol. SAC-5, pp. 128-137, Feb. 1987.

[8] T. Rappaport, S. Seidel, and R. Singh, "900-Mhz multipath propagation measurements for U.S. digital cellular radiotelephone," IEEE Trans. Veh. Technol., vol. 39, pp. 132-139, May 1990.

[9] J. G. Proakis, Digital Communications. New York: McGraw-Hill, 1989.

[10] Z. Zvonar and D. Brady, "Optimum detection in asynchronous multipleaccess multipath Rayleigh fading channels," in Proc. 26th Annual Conf. Infor. Sci. Systems, Mar. 1992

[11] M. K. Varanasi and S. Vasudevan, "Multiuser detectors for synchronous CDMA communication over nonselective Rician fading channels," IEEE Trans. Commun., vol. 42, pp. 711-722, Feb. 1994.

[12] S. Y. Miller and S. C. Schwartz, "Parameter estimation for asynchronous multiuser communication," in Proc. 1989 Conf. Information Sci. Systems, 1989, pp. 294-299.

[13] U. Fawer and B. Aazhang, "A multiuser receiver for code division multiple access communications over multipath channels," IEEE Trans. Commun. vol. 43, pp. 1556-1565, Feb./Mar./Apr. 1995.

[14] S. E. Bensley and B. Aazhang, "Maximum likelihood estimation of a single user's delay for code division multiple access communication systems," in Proc. Inform. Sciences Systems, 1994, also accepted for publication in IEEE Trans. Commun..

[15] D. H. Johnson and D. E. Dudgeon, Array Signal Processing: Concepts and Techniques. Englewood Cliffs, NJ: Prentice-Hall, 1993.

[16] E. G. Ström, S. Parkvall, S. L. Miller, and B. E. Ottersen, "Propagation delay estimation in asynchronous direct-sequence code-division multiple access systems," submitted to IEEE Trans. Commun.

[17] M. Wax and T. Kailath, "Detection of signals by information theoretic criteria," IEEE Trans. Acoustic, Speech, Signal Processing, vol. ASSP33, pp. 387-392, Apr. 1985.

[18] R. Roy and T. Kailath, "ESPRIT-Estimation of signal parameters via rotational invariant techniques," IEEE Trans. Acoust., Speech, Signal Processing, vol. 37, no. 7, pp. 984-995, July 1989.

[19] A.-J. van der Veen, E. F. Deprettere, and A. L. Swindlehurst, "Subspacebased signal analysis using singular value decomposition," Proc. IEEE, vol. 81 , pp. 1277-1308, Sept. 1993.

[20] R. Muirhead, Aspects of Multivariate Statistical Theory. New York: Wiley, 1982.

[21] K. Sharman and T. S. Durrani, "A comparative study of modern eigenstructure methods for bearing estimation-A new high performance approach," in Proc. 25th IEEE Conf. Dec. Contr., Athens, Greece, Dec. 1986, pp. 1737-1742.

[22] R. O. Schmidt, "A signal subspace approach to multiple emitter location and spectral estimation," Ph.D. dissertation, Stanford University, Stanford, CA, 1981. 
[23] R. K. Madyastha and B. Aazhang, "Multiuser receivers for CDMA communication systems using antenna arrays," in 32nd Ann. Allerton Conf. Comm. Contr, and Comp., Monticello, IL., Sept. 28-30, 1994.

[24] P. Stoica and A. Nehorai, "Statistical efficiency study of direction estimation methods part I: Analysis of MUSIC and preliminary study of MLM," in Advances in Spectrum Analysis and Array ProcessingVolume II, S. Haykin, Ed. Englewood Cliffs, NJ: Prentice-Hall, 1991, pp. 263-305.

[25] R. Lupas and S. Verdú, "Near-far resistance of multiuser detectors in asynchronous channels," IEEE Trans. Commun., vol. 38, pp. 496-508, Apr. 1990.

[26] R. D. DeGroat, "Noniterative subspace tracking," IEEE Trans. Signal Processing, vol. 40, pp. 571-577, Mar. 1992.

[27] G. W. Stewart, "An updating algorithm for subspace tracking," IEEE Trans. Signal Processing, vol. 40, pp. 1535-1541, June 1992.

[28] B. Yang, "Subspace tracking based on the projection approach and the recursive least squares method," in Proc. ICASSP, 1993, vol. IV, pp. $145-148$.

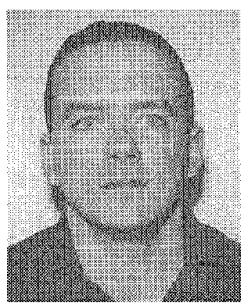

Stephen E. Bensley was born in Boston, MA, on July 4, 1966. He received the B.S. and M.S. degrees in electrical engineering from Rice University, Houston, TX, in 1987 and 1994, respectively.

$\mathrm{He}$ is currently working on the performance anal ysis of packet switching and ATM networks for Cabletron Systems in Durham, NH.

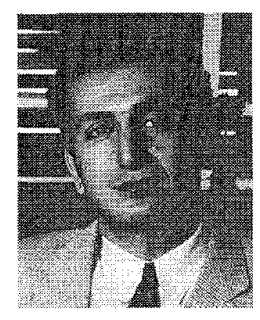

Behnaam Aazhang (S'81-M'85-SM'91) was born in Bandar Anzali, Iran, on December 7, 1957. He received the B.S. (with highest honors), M.S., and Ph.D. degrees in electrical and computer engineering from the University of Illinois at UrbanaChampaign, in 1981, 1983, and 1986, respectively.

From 1981 to 1985, he was a Research Assistant in the Coordinated Science Laboratory, University of Illinois. In August 1985, he joined the faculty of Rice University, Houston, TX, where he is now a Professor in the Department of Electrical and Computer Engineering. He has been a Visiting Professor at IBM Federal Systems Company, Houston, TX, the Institut für Kommunikationstechnik at the Swiss Federal Institute of Technology (ETH), Zürich, Switzerland, the Telecommunications Laboratory at University of Oulu, Oulu, Finland, and at the US Air Force Phillips Laboratory, Albuquerque, NM. His research interests are in the areas of communication theory, information theory, and their applications with emphasis on multiple access communications; cellular mobile radio communications; optical communication networks; and efficient simulation of stochastic systems. He is currently serving as the Editor for Spread Spectrum Networks of IEEE TRANSACTIONS ON COMMUNiCATIONS. and as the Treasurer of IEEE Information Theory Society. He has served as the Secretary of the Information Theory Society 1990-1993 and as the Publications Chairman of the 1993 IEEE International Symposium on Information Theory, San Antonio, TX.

Dr. Aazhang is a recipient of the Alcoa Foundation Award 1993, the NSF Engineering Initiation Award 1987-1989, and the IBM Graduate Fellowship 1984-1985. He is a member of Tau Beta Pi and Eta Kappa Nu. 